\title{
Astrometric observations of 51 Peg and Gliese 623 at Pulkovo observatory with $65 \mathrm{~cm}$ refractor
}

\author{
N.A. Shakht \\ Central (Pulkovo) Astronomical Observatory, \\ Russian Academy of Sciences, St.Petersburg, Russia \\ email: shakht@gao.spb.ru
}

\begin{abstract}
The photographic observations of 51 Peg with the known planetary companion which has been discovered on the basis of radial velocities (Mayor and Quelos 1995), are being performed at Pulkovo since 1995 by means of $65 \mathrm{~cm}$ refractor. So far 46 plates with 170 individual positions have been obtained. The mean error of one exposure is $0 .{ }^{\prime \prime} 031$ and the error of one plate is $0 .{ }^{\prime \prime} 020$. The external error of one plate or the error of unit weight is $0 . .^{\prime \prime} 033$, while the error of one yearly position equals to $0 . .^{\prime \prime} 010$. The aim of our observations is to investigate whether this star has some satellites of low mass (stellar or substellar) with periods of rotation from some years to a decade, or more. At present we have the possibility to study its motion over a time span of 8 years and to learn of probable perturbations due to the presence of possible satellites with the periods $0.5-6.0$ years and with masses more than $0.2 M_{\odot}$. The absence of satellites with such periods and masses has been shown by means of our observations. The results are compared with the Pulkovo series of the star Gliese 623, whose satellite with the period of $3.76 \mathrm{yr}$ and mass of $0.09 M_{\odot}$, has been confirmed by observations.
\end{abstract}

Keywords. Photographic observations, individual: 51 Peg, Gliese 623, planetary satellites

\section{Introduction}

51 Peg $\left[m_{\mathrm{vis}}=5 .^{m} 5, G 5 V, \alpha_{(2000.0)}=22^{h} 57 .{ }^{m} 5, \delta_{(2000.0)}=+20^{\circ} 46^{\prime}, \pi=0^{\prime \prime} .074\right]$ with the known planetary satellite (Mayor and Quelos 1995) is inluded the Pulkovo program of single and double stars with suspected unseen components. The plate measurements have been made with Pulkovo automatic machine "Fantasy". The relative positions with respect to six stars with small proper motions, obtained in the period 1995-2000, have been published in Shakht et al. (2002). Our aim was to check the residuals $\mathrm{O}-\mathrm{C}$ remained after the excluding of proper motion and parallactic displacement of this star.

\section{Limitations of our astrometric observations}

It is rather straightforward to determine the limits for the periods and masses of the components whose gravitational influence we could reveal from our observations with Pulkovo $65 \mathrm{~cm}$ refractor and from the comparison of the precision of observations with the gravitational influence of real and suspected satellites of the star.

A number of selected stars from our program are presented in Table 1 . Here $D$ denotes the distance of the star from the Sun in parsecs, $M$ is its mass in $M_{\odot}, \sigma$ is the error of the mean yearly position given in milliarcsecs. R1-R5, expressed in milliarcsecs, are the angular displacements of the principal star with respect to the centre of mass due to a 
satellite with corresponding mass and period of rotation (the third and fourth lines in the columns 7-11). $\Delta T$ is the duration of regular observations expressed in years (61 Cyg was observed with $65 \mathrm{~cm}$ refractor for 42 years and with normal astrograph for more than 100 years).

The angular displacements which can be detected with $65 \mathrm{~cm}$ refractor are marked in bold. The displacements which have been discovered in our series are marked by asterisks. In this case, we suppose that they are caused by suspected dark satellites of low masses. The displacements caused by real satellites, confirmed for Gliese 623 and discovered for $\delta$ Gem on the basis of Pulkovo observations (Shakht 1995, 1997, 2000) are marked by double asterisks. R1 corresponds to the influence of a satellite with the Jupiter-like mass and period.

Using Scargle's method of periodograms (Black and Scargle 1982) and taking into account the specific distribution of $51 \mathrm{Peg}$ observations in time and the precision of our observations, we conclude that our series allow to discover the possible perturbations from the satellites with periods from 0.5 to 6.0 years and with amplitudes more than $0 .{ }^{\prime \prime} 020$ and hence with masses more than $0.2 M_{\odot}$.

Table 1. Detectable displacements for Pulkovo program stars

\begin{tabular}{|c|c|c|c|c|c|c|c|c|c|c|}
\hline | 1 & 2 & 3 & $4 \mid$ & 5 & 6 & 7 & 8 & 9 & $10 \mid$ & 11 \\
\hline $\mathrm{No}$ & Name & $\begin{array}{r}D \\
{[\mathrm{pc}]}\end{array}$ & $\begin{array}{r}M \\
{\left[M_{\odot}\right]}\end{array}$ & $\begin{array}{l}\Delta T \\
{[\mathrm{yr}]}\end{array}$ & $\begin{array}{r}\sigma \\
{[\mathrm{mas}]}\end{array}$ & \begin{tabular}{|r}
$\mathrm{R} 1$ \\
0.001 \\
12
\end{tabular} & \begin{tabular}{|r}
$\mathrm{R} 2$ \\
0.01 \\
15
\end{tabular} & $\begin{array}{r}\mathrm{R} 3 \\
0.01 \\
3.5\end{array}$ & $\begin{array}{l}\mathrm{R} 4 \\
0.1 \\
3.5\end{array}$ & $\begin{array}{l}\text { R5 } \\
0.2 \\
6.0\end{array}$ \\
\hline 1 & Lal21185 & 2.5 & 0.33 & 32 & 16 & 4 & 50 & 18 & 160 & 400 \\
\hline 2 & $61 \mathrm{C}$ & 3. & 0.70 & 42 & 7 & 2 & $* 20$ & *8 & 65 & 200 \\
\hline 3 & ADS11632 & 3.5 & 0.34 & 36 & 12 & 4 & *34 & $* 13$ & 110 & 280 \\
\hline 4 & Gliese 623 & 7.2 & 0.34 & 20 & 8 & 15 & 16 & 6 & $* * 55$ & 140 \\
\hline 5 & $51 \mathrm{Peg}$ & 13.5 & 1.0 & 8 & 10 & 0.4 & 4 & 2 & 16 & 44 \\
\hline 6 & $\delta$ GemA & 16.4 & 1.8 & 32 & 18 & 0.2 & 2 & 1 & 9 & $* * 25$ \\
\hline
\end{tabular}

\section{Results}

At the first stage, we have obtained the values of the parallax and proper motion of $51 \mathrm{Peg}$ and then the residuals $\mathrm{O}-\mathrm{C}$ have been analysed. In Table 2, the relative proper motion and parallax of $51 \mathrm{Peg}$ with their errors are given. The errors of unit weight $\sigma_{x}$ and $\sigma_{y}$ obtained by least-squares solution are given too.

Table 2. Relative proper motion and parallax of $51 \mathrm{Peg}$

$$
\begin{array}{|c|c|c|c|}
\hline \mu_{x} & 0 .{ }^{\prime \prime} 2159 \pm 0 . .^{\prime \prime} 0030 & \mu_{y} & 0 . .^{\prime \prime} 0695 \pm 0 . .^{\prime \prime} 0032 \\
\pi_{x} & 0 .{ }^{\prime \prime} 0645 \pm 0 .{ }^{\prime \prime} 0083 & \pi_{y} & 0 .{ }^{\prime \prime} 0721 \pm 0 .{ }^{\prime \prime} 0134 \\
\sigma_{x} & \pm 0^{\prime \prime} .032 & \sigma_{y} & \pm 0 .{ }^{\prime \prime} 034 \\
\hline
\end{array}
$$

One can see that the precision is insufficient for the direct determination of parallaxes so that we excluded the parallactic displacements from the relative positions of star using the catalog values of parallaxes. Moreover, one can notice that the errors for $\pi_{x}$ and for $\pi_{y}$ are of comparable size, because this star is close to the pole of the ecliptique. With larger observational material we can use these values for the estimation of the influence of possible satellites with low masses, if we would find some correlations between perturbations and parallactic displacements. 
We can compare $\mathrm{O}-\mathrm{C}$ obtained for $51 \mathrm{Peg}$ with the $\mathrm{O}-\mathrm{C}$ obtained for Gliese 623 $\left[10 .^{m} 5, M 3, \alpha_{(2000.0)}=16^{h} 24^{m} .0, \delta_{(2000.0)}=+48^{\circ} 21^{\prime}, \pi=0 .^{\prime \prime} 138\right]$. The latter star has been observed at Pulkovo since 1979 (Shakht 1995, 1997) with the same precision as 51 Peg. Residuals $\mathrm{O}-\mathrm{C}$ (see Figs. 1 and 2). have been obtained in the same way, that is after the exclusion of proper motion and parallactic displacements.
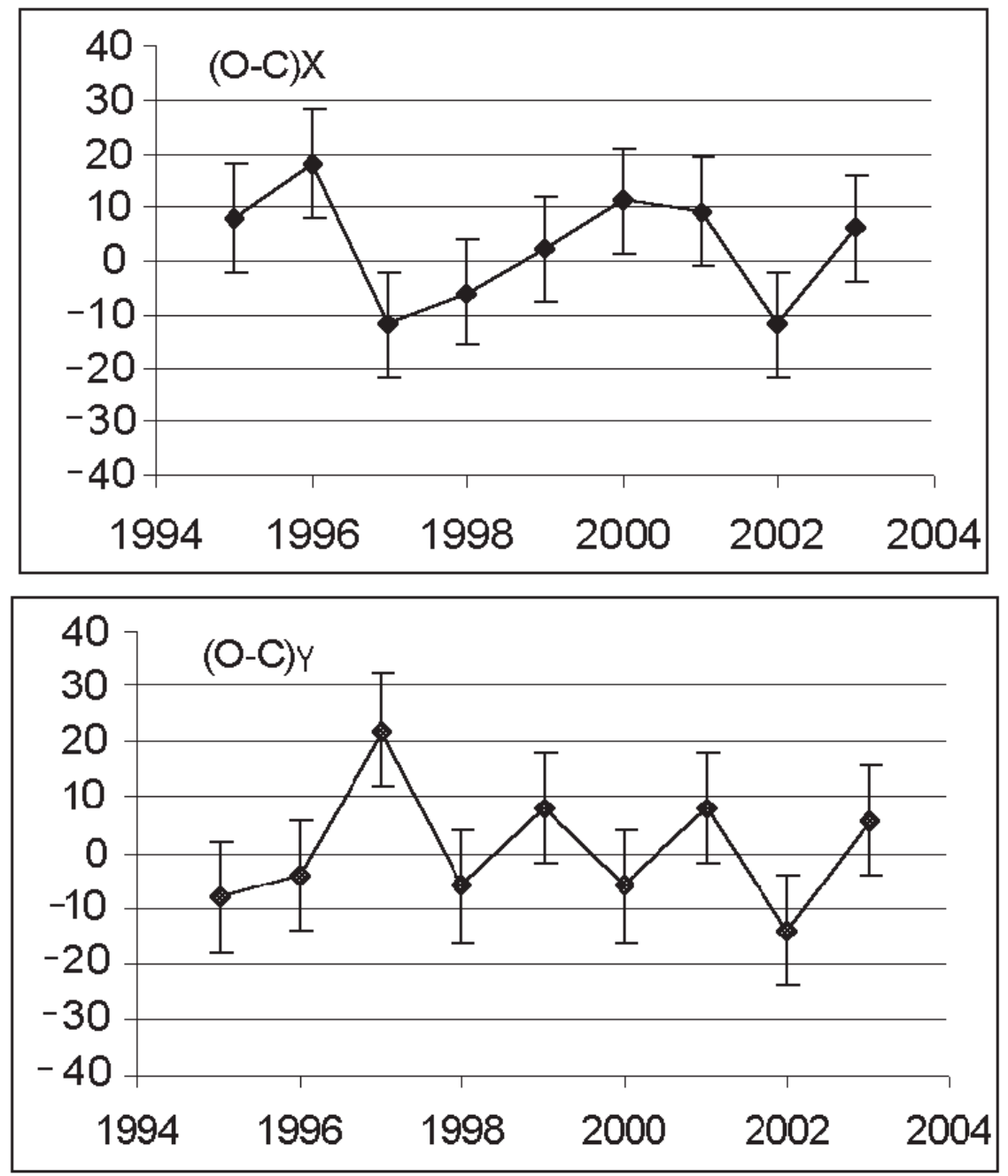

Figure 1. Residuals in the motion of $51 \mathrm{Peg}$ (in mas).

The comparison has shown that the error of unit weight for Gliese 623 calculated on the basis of the residuals $\mathrm{O}-\mathrm{C}$, equals $0 .{ }^{\prime \prime} 042$ for both coordinates because it contains the orbital motion of the photocenter, whereas the corresponding error for 51 Peg equals to $0 .{ }^{\prime \prime} 033$.

Fig. 3 shows the power spectrum $S(\omega)$, obtained on the basis of residuals in the motion of $51 \mathrm{Peg}$ (I) and for Gliese 623 (II). The latter is presented for comparison. The frequency is equal to 0.26 , and corresponds to the period of 3.76 years for a satellite of the mass $0.09 M_{\odot}$, which belongs to Gliese 623 and which has been discovered by astrometric method (Lippincott and Borgman 1978) and confirmed by means of other astrometric 

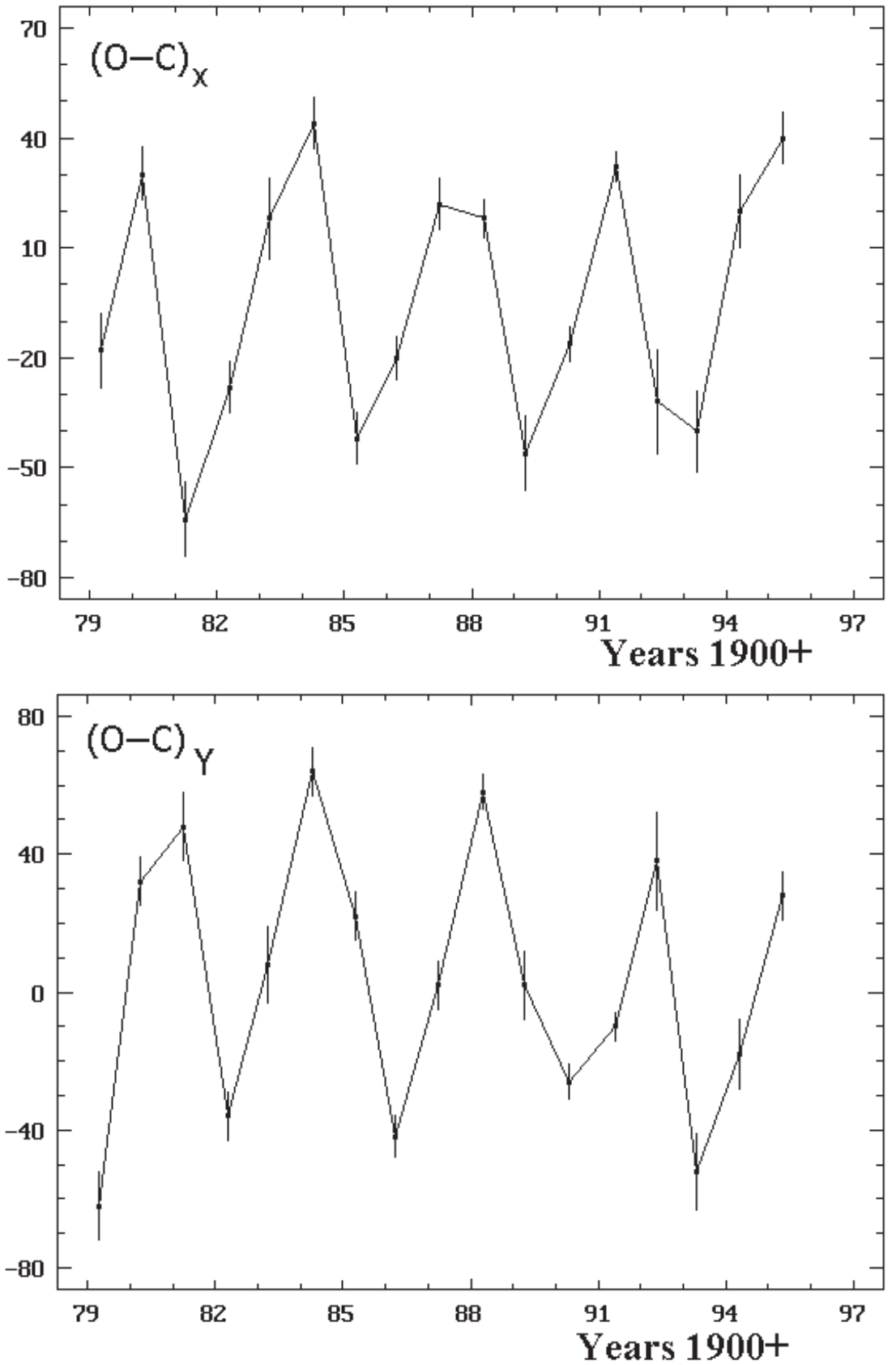

Figure 2. Residuals in the motion of Gliese 623 (in mas).

observations, including Pulkovo data. One can also see that $S(\omega)$ for $51 \mathrm{Peg}$ does not show any noticeable frequencies and periods.

We used the method of the periodogram by Scargle to study the behavior of residuals and for the calculations of possible perturbations from the satellites with periods and masses shown in Table 3. Table 3 gives calculated displacements of the photocenter of 51 Peg caused by theoretically possible satellites. 


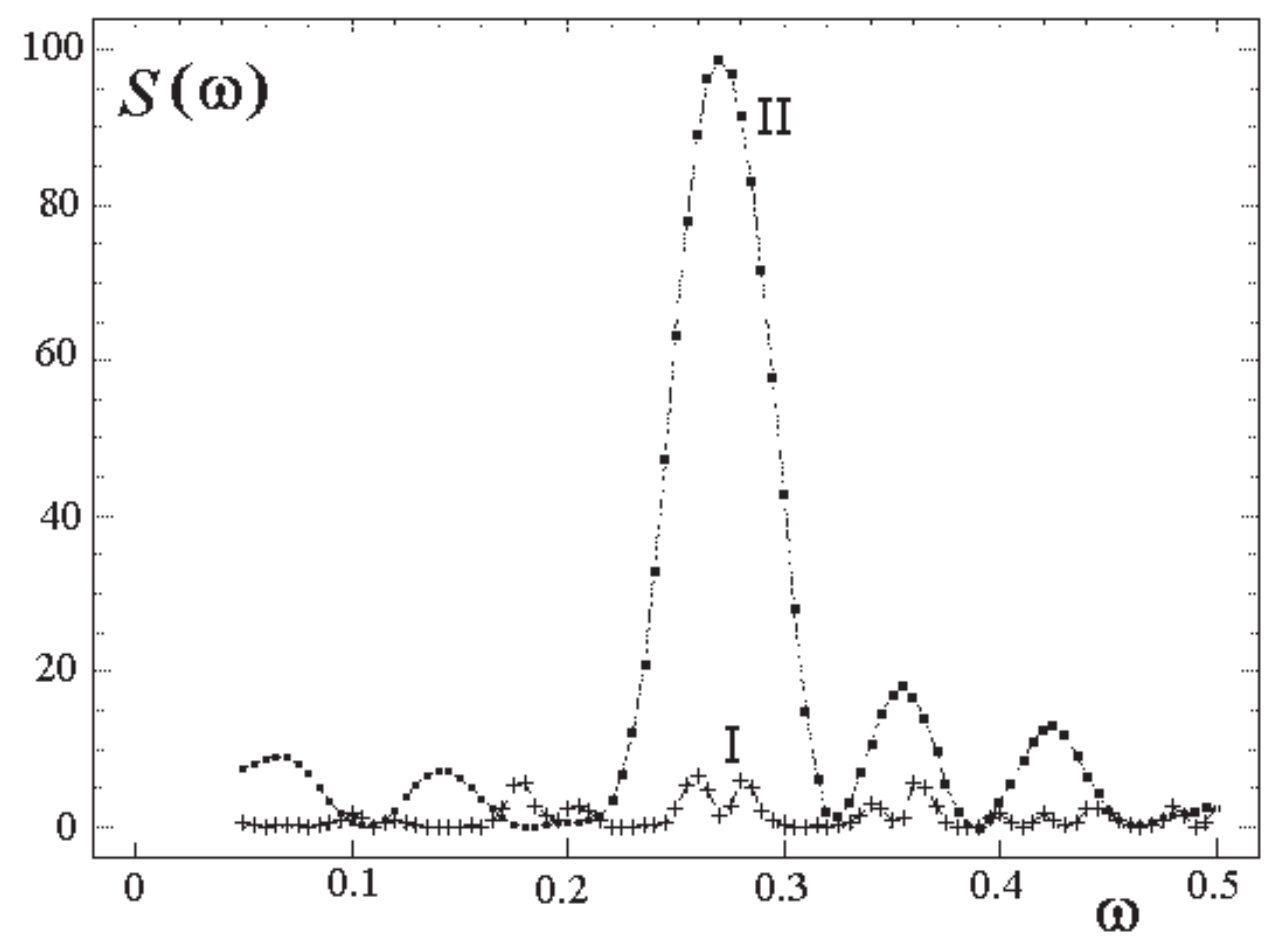

Figure 3. The periodogram obtained on the basis $\mathrm{O}-\mathrm{C}$ in the motion of $51 \mathrm{Peg}$ (crosses) and in the motion of Gliese 623 (dots). The ordinate is the power $S(\omega)$, where $\omega=1 / P$ and $P$ is period in years. The great peak corresponds to the period $3.76 \mathrm{yr}$ of the satellite of Gliese 623 .

Table 3. Calculated displacements in the motion of 51 Peg due to the possible satellites

\begin{tabular}{|l|r|r|r|r|r|}
\hline$P[\mathrm{yr}]$ & $1^{y} .5$ & $2^{y} .5$ & $3^{y} .0$ & $3^{y} .5$ & $6^{y} .0 \mid$ \\
\hline $0.5 M_{\odot}$ & $\mathbf{0}^{\prime \prime} . \mathbf{0 3 7}$ & $\mathbf{0}^{\prime \prime} . \mathbf{0 5 0}$ & $\mathbf{0}^{\prime \prime} . \mathbf{0 6 0}$ & $\mathbf{0}^{\prime \prime} . \mathbf{0 6 5}$ & $\mathbf{0}^{\prime \prime} . \mathbf{0 9 5}$ \\
0.4 & $\mathbf{3 0}$ & $\mathbf{4 0}$ & $\mathbf{5 0}$ & $\mathbf{5 5}$ & $\mathbf{7 8}$ \\
0.3 & $\mathbf{2 4}$ & $\mathbf{3 5}$ & $\mathbf{4 0}$ & $\mathbf{4 5}$ & $\mathbf{6 2}$ \\
0.2 & 17 & $\mathbf{2 4}$ & $\mathbf{2 7}$ & $\mathbf{3 0}$ & $\mathbf{4 4}$ \\
0.1 & 9 & 13 & 14 & 16 & 23 \\
0.08 & 7 & 10 & 12 & 13 & 18 \\
\hline
\end{tabular}

Table 4. Parameters of the possible satellites of $51 \mathrm{Peg}$

\begin{tabular}{l}
\hline$P[\mathrm{yr}] \quad|1.5| 2.5|3.0| 3.5|3.5| 4.0|4.0| 6.0|6.0|$ \\
\hline$M_{\odot} \quad|0.2| 0.2|0.2| 0.1|0.2| 0.2|0.1| 0.2|0.08|$ \\
\hline$A$ in a.e. $|1.2| 1.6|1.8| 2.2|2.0| 2.2|2.4| 3.0|3.1|$
\end{tabular}

In the Table 4 the parameters of the possible satellites of 51 Peg accessible to Pulkovo $65 \mathrm{~cm}$ refractor after 12 years of observations are given. Among these there are satellites with masses less than $0.1 M_{\odot}$. 


\section{Conclusions}

We present our first regular series of astrometric observations of 51 Peg performed by means of a long-focus astrograph, on the basis of which we were able to analyse a limited range of proposed periods and masses of possible satellites of $51 \mathrm{Peg}$. So far no perturbations due to the presence of possible satellites with the periods $0.5-6.0$ years and with masses more than $0.2 M_{\odot}$ were found. Nevertheless, we have photographic plates which permit to continue the observations of this star. The precision of the observations and measurements by automatic Pulkovo complex "Fantasy" will provide the possibility to estimate the influence of satellites with masses less than $0.1 M_{\odot}$, near to the limit mass of the brown dwarfs $0.08 M_{\odot}$, and over a rather large range of rotation periods.

One of the important problems of the Pulkovo $65 \mathrm{~cm}$ refractor remains the necessity to have large field CCD to be used for observations in the frame of our program.

\section{References}

Black D.C. and Scargle J.D 1982, Astron. J. 263, (2), 854

Lippincott S.L. and Borgman E.R. 1978, Publ. Astron. Soc. Pacific, 90, 226

Mayor M. and Quelos D. 1995, Nature, 378, 355

Shakht N.A. 1995, Proc. Symp. 166 IAU, 359

Shakht N.A. 1997, Astr. Astroph. Trans., 13, 327

Shakht N.A. 2000, Izvestiya GAO 214, 77 (in Russian).

Shakht N.A., Grosheva E.A., Kisselev A.A., Polyakov E.V. and Rafalsky V.B. 2002, Izvestiya $G A O, 216,363$ (in Russian) 